\title{
Airflow limitation, lung volumes and systemic inflammation in a general population
}

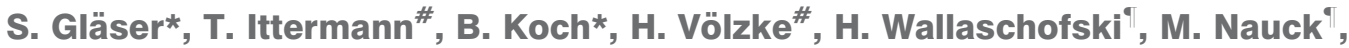 \\ C. Warnke*, C. Vogelmeier ${ }^{+}$, H. Schulz ${ }^{\S}$, S.B. Felix*, R. Ewert* and C. Schäper*
}

ABSTRACT: Although several levels of evidence have suggested an association between systemic inflammation and spirometric lung volumes, data addressing the potential interrelationship between airflow limitation and inflammatory markers are sparse and remain controversial.

Potential associations between high-sensitivity C-reactive protein (hsCRP), fibrinogen and lung function were investigated in 1,466 individuals aged 25-85 yrs, representing a general population. Within this cross-sectional population, data on body plethysmography, spirometry, helium dilution and diffusing capacity of the lung for carbon monoxide ( $D L, C O)$ were analysed.

After adjustment for potential confounding factors, such as smoking, obesity and cardiorespiratory fitness, there was an inverse association of hsCRP with forced expiratory and static lung volumes. In neither apparently healthy nor the entire population was inflammation associated with airflow limitation in central airways. In smokers only, higher hsCRP and fibrinogen were associated with an impaired DL,Co.

This study shows that higher levels of hsCRP are associated with decreased lung volumes in a general population over a wide age range. A consistent interrelationship of central airflow limitation and inflammation was not verifiable. Smoking is related to an impaired $D L, C O$ in association with an increase in systemic inflammation.

KEYWORDS: C-reactive protein, diffusing capacity, inflammation, lung function, population-based studies

everal levels of evidence have consistently found significant association between markers of systemic inflammation and spirometric lung volumes [1-4]. Moreover, systemic inflammation as quantified by serum C-reactive protein (CRP) or fibrinogen is related to the longitudinal decline in spirometric lung volumes [2]. So far, the interrelationship between reduced lung volumes and inflammation remains poorly understood. CRP, as one of the most widely used serum markers of systemic inflammation, is associated with increased mortality and incidental adverse cardiovascular events independently of potential interfering factors [5]. Correspondingly, decreased lung volumes have also been shown to be consistently related to all causes of mortality [6, 7]. Thus, systemic inflammation has been discussed as one potential pathophysiological link between lung volumes and excess mortality [2].

Although the studies investigating the potential association of inflammation and lung volumes are numerous, each of these investigations had limitations. First, some of these studies did not use high-sensitivity (hs)CRP and may have missed the influence of low-grade inflammation. Secondly, the majority of studies investigated pre-selected specific age decades, restricted either to young, middle-aged or elderly volunteers. Hence, none of these studies represented a general population. Thirdly, most studies are based on spirometric evaluations only and may be strongly biased by the volunteers' compliance.

In contrast to the evident association between lung volumes and inflammatory markers, investigations relating systemic inflammation to airflow limitation have shown controversial results. In general, diseases characterised by airflow limitation, such as chronic obstructive pulmonary disease (COPD), appear to be associated with systemic inflammation [8, 9]. Even if patients with COPD have increased CRP or fibrinogen compared with healthy controls, a continuous correlation between the level of inflammation and the extent of airflow limitation has not been
AFFILIATIONS

*Dept of Internal Medicine B,

Cardiology, Intensive Care,

Pulmonary Medicine and Infectious Diseases,

\# Institute for Community Medicine, SHIP/Clinical-Epidemiological

Research,

-Institute of Clinical Chemistry and Laboratory Medicine, University of Greifswald, Greifswald

${ }^{+}$Dept of Pulmonary Medicine, University of Marburg, Marburg, and ${ }^{\S}$ Helmholtz Zentrum München, German Research Center for Environmental Health, Institute of Epidemiology I, Neuherberg, Germany.

CORRESPONDENCE

S. Gläser

Medical Faculty of the Ernst-MoritzArndt University

Dept of Internal Medicine B,

Cardiology, Intensive Care, Pulmonary Medicine and Infectious Diseases Friedrich-Loeffler-Str. 23 D-17475 Greifswald Germany E-mail: glaeser.sven@gmail.com

Received:

Jan 192011

Accepted after revision: May 172011

First published online: June 302011 
shown and the relationship appears to be influenced by comorbidities, obesity and physical activity [9].

This investigation aimed to assess a potential link between low-grade systemic inflammation, quantified by hsCRP and fibrinogen, and lung function, based on a highly standardised setting within a population-based study representing a general population aged 25-85 yrs in the north-east of Germany: the Study of Health in Pomerania (SHIP). The main hypothesis to be investigated is that systemic inflammation, even within the low-grade range, is associated with airflow limitation, lung volumes and diffusing capacity, independently of potentially confounding factors, such as obesity, physical activity, cardiopulmonary diseases or smoking. In addition to spirometry, body plethysmography, helium dilution for measurement of alveolar volume and diffusing capacity of the lung for carbon monoxide $(D \mathrm{~L}, \mathrm{CO})$ were applied to ensure additional physiological insight into the hypothesised link.

\section{METHODS}

\section{Study population}

The SHIP study is a population-based investigation in West Pomerania, a region in the north-eastern part of Germany. Study details are given elsewhere [10]. In brief, a sample from the population aged 20-79 yrs was drawn to be evaluated during baseline SHIP-0 from 1997 to 2001. Between March 2002 and September 2006, the 5-yr follow-up examinations (SHIP-1) were performed and comprised 3,300 participants (1,711 females). The study was reviewed by a board of independent scientists and approved by the Ethics Committee of the University of Greifswald (Greifswald, Germany). All participants provided written informed consent.

Blood samples to measure inflammatory markers were obtained during SHIP-0 and SHIP-1. Lung function examination, including body plethysmography and $\mathrm{DL}, \mathrm{CO}$, was offered to all participants and was performed on 1,809 individuals (885 males) during SHIP-1. The mean (range) time interval between core examination of SHIP-1 and lung function examination was 0.6 (0.0-2.5) months. Of these, we excluded 275 participants because of steroid intake or chronic use of nonsteroidal anti-rheumatics and 29 participants because of missing data. Levels of hsCRP $>10 \mathrm{mg} \cdot \mathrm{L}^{-1}$, indicative of acute and active infection, systemic inflammatory processes or physical trauma, were defined as an additional exclusion criterion $(n=38)$ [11]. In total, data from 1,466 participants were analysed.

\section{Pre-exercise diagnostics}

Sociodemographic and medical characteristics were assessed by computer-assisted personal interviews. Previous history of diseases was based on self-reported physician's diagnosis. According to tobacco consumption, participants were categorised into current $\left(\geqslant 1\right.$ cigarettes $\cdot$ day $\left.^{-1}\right)$, former and nonsmokers. Data on medication were collected using the anatomical therapeutic chemical (ATC) code [12]. Height and weight were measured for the calculation of the body mass index (BMI; in $\left.\mathrm{kg} \cdot \mathrm{m}^{-2}\right)$. Waist circumference was measured to the nearest $0.1 \mathrm{~cm}$ using an inelastic tape midway between the lower rib margin and the iliac crest in the horizontal plane in standing position. The measurement was taken at the level of the narrowest part of the waist. Physical activity was assessed by standardised interview $\left(<2\right.$ or $\geqslant 2 \mathrm{~h} \cdot$ week $\left.^{-1}\right)$. All interviews and measures were obtained at the time of lung function examination.

\section{Blood sampling}

For the laboratory examinations, nonfasting blood samples were drawn between 07:00 $\mathrm{h}$ and 16:00 $\mathrm{h}$, during baseline examinations of SHIP-0 and SHIP-1. hsCRP was determined immunologically on a Behring Nephelometer II with commercially available reagents from Dade Behring (Dade Behring, Eschborn, Germany). Plasma fibrinogen concentrations were assayed according to the method of CLAUSS [13] using an Electra 1600 analyser (Instrumentation Laboratory, Barcelona, Spain).

To adjust DL,CO for the potential influence of blood haemoglobin levels, capillary blood from the ear lobe was drawn on the day of lung function testing. The capillary blood was collected in a $55-\mu \mathrm{L}$ capillary (Clinitubes $\mathbb{R}$; Radiometer, Copenhagen, Denmark) and immediately transferred to the blood gas and haemoglobin analyser (Radiometer ABL 510; Radiometer) to determine haemoglobin concentration.

\section{Procedure and variables}

The lung function examinations were conducted using a body plethysmograph equipped with a pneumotachograph (MasterScreen Body/Diff.; VIASYS Healthcare, JAEGER, Hoechberg, Germany), which meets American Thoracic Society (ATS) criteria [14]. All calibrations and tests were carried out in the following order in accordance with European Respiratory Society (ERS) and ATS recommendations [15]: 1) static lung volume determination using variable-pressure body plethysmography; and 2) forced spirometry. The procedures were conducted with the subject in a sitting position and wearing a nose-clip. Pressure-flow and pressure-pressure diagrams were registered to obtain the specific and total airway resistance (Raw). Functional residual capacity (FRCpleth) was measured during expiration, breathing against a shutter near FRC. A series of gentle pants were obtained (three to five panting manoeuvres) against the shutter, followed by deep expiration to measure expiratory reserve volume (ERV) and inspiratory slow vital capacity (VCslow). The residual volume (RV) was obtained by subtracting the ERV from the FRCpleth. The total lung capacity (TLC) was computed from RV and VCslow, and RV/TLC was calculated as a percentage. Forced spirometric manoeuvres were obtained to measure forced vital capacity (FVC), forced expiratory volume in $1 \mathrm{~s}$ (FEV1) and the maximal expiratory flow at $75 \%, 50 \%$ and $25 \%$ of FVC (MEF $75 \%$, MEF50\% and MEF25\%, respectively). At least three acceptable attempts at fulfilling reproducibility criteria for all variables according to the ATS/ERS guidelines were required (e.g. FRC variability $\leqslant 5 \%$; Raw variability $\leqslant 5 \%$;EV1, VCslow and FVC variability $<150 \mathrm{~mL})[15,16]$.

After a break of $\geqslant 3 \mathrm{~min}$, the assessment of $D \mathrm{~L}, \mathrm{CO}$ by a singlebreath (SB) manoeuvre with $\mathrm{CO}$ was applied [17]. In parallel, alveolar volume $(V \mathrm{~A})$ was measured using helium dilution [15]. DL,CO-SB was corrected for current haemoglobin $\left(D \mathrm{~L}, \mathrm{CO}_{\mathrm{C}^{-}}\right.$ $\mathrm{SB})$ and $V \mathrm{~A}\left(D \mathrm{~L}, \mathrm{CO}_{\mathrm{C}}-V \mathrm{~A}\right)$.

\section{Statistical analysis}

Data on quantitative characteristics are expressed as median and interquartile range. Data on qualitative characteristics are expressed as percentages or absolute numbers, as indicated. Differences between males and females were tested by 
Wilcoxon test for continuous data and by Chi-squared test for categorical data. Linear regression models were used to assess the association between inflammatory markers and pulmonary parameters. Fractional polynomials were applied to explore and graph nonlinear associations [18]. The dose-response relationship was found using fractional polynomials up to degree 2 with all possible combinations of powers selected from the set $(-2,-1,-0.5,0,0.5,1,2,3)$ and comparing them using the log likelihood to determine the best-fitting model. If none of the fractional polynomial models fitted the data significantly better than the linear model, linear regression was applied. All models were adjusted for age, sex, smoking status, BMI, heart failure (self-reported physician's diagnosis, echocardiographic evidence of left-ventricular or valvular dysfunction and/or in accordance with the Rotterdam Study heart failure definition (two-step approach, including the presence of shortness of breath at rest or on exertion, ankle oedema and pulmonary crepitations in addition to evidence of cardiac disease) [19]), COPD (self-reported physician's diagnosis), asthma (self-reported physician's diagnosis), and time between core and lung function examination. For sensitivity testing, BMI was substituted by waist circumference, height and weight. For additional sensitivity analyses, participants with the following were excluded: evidence of coronary artery disease (myocardial infarction, signs of ischaemia in ECG); restrictive lung disease (TLC below the fifth percentile and FEV1/VCslow ratio above the fifth percentile); and airflow limitation (FEV1/FVC and/or FEV1/VCslow ratios below the fifth percentile) $[20,21]$. In addition, separate analyses were obtained for subjects with the following results below the fifth percentile: FEV1/FVC and/or FEV1/VCslow ratios, TLC and $D \mathrm{~L}, \mathrm{CO}_{\mathrm{c}}-V \mathrm{~A}$. For this purpose, the reference equation for $D \mathrm{~L}, \mathrm{CO}_{\mathrm{c}^{-}}$ $V A$ was assessed in accordance with the recommendations of the ERS [22]. Based on the methodology of lung function reference assessment within this population, the following reference equations were applied [21]:

$$
\begin{aligned}
& D \mathrm{~L}, \mathrm{CO}_{\mathrm{c}}-V \mathrm{~A}_{(5 \text { th percentile })}= \\
& 1.6993-(0.0074 \times \text { age })+(0.0595 \times \text { sex })-(0.001 \times \text { height }) \\
& \mathrm{TLC}_{(5 \text { th percentile males })}= \\
& -11.2726+(0.0027 \times \text { age })+(0.0964 \times \text { height }) \\
& \mathrm{TLC}_{(5 \text { th percentile females })}= \\
& -4.4949+(0.0031 \times \text { age })-\left(0.000045 \times \text { age }^{2}\right)+(0.0545 \times \text { height })
\end{aligned}
$$

Height was expressed in $\mathrm{cm}$, and for sex, males were given a value of 1 , females 0 . These reference equations were compared with those provided by QUANJER et al. [23] and COTES et al. [24].

As recommended for epidemiological studies with missing data, multiple imputations of missing data via chained equations

\section{TABLE 1 Characteristics of the study population}

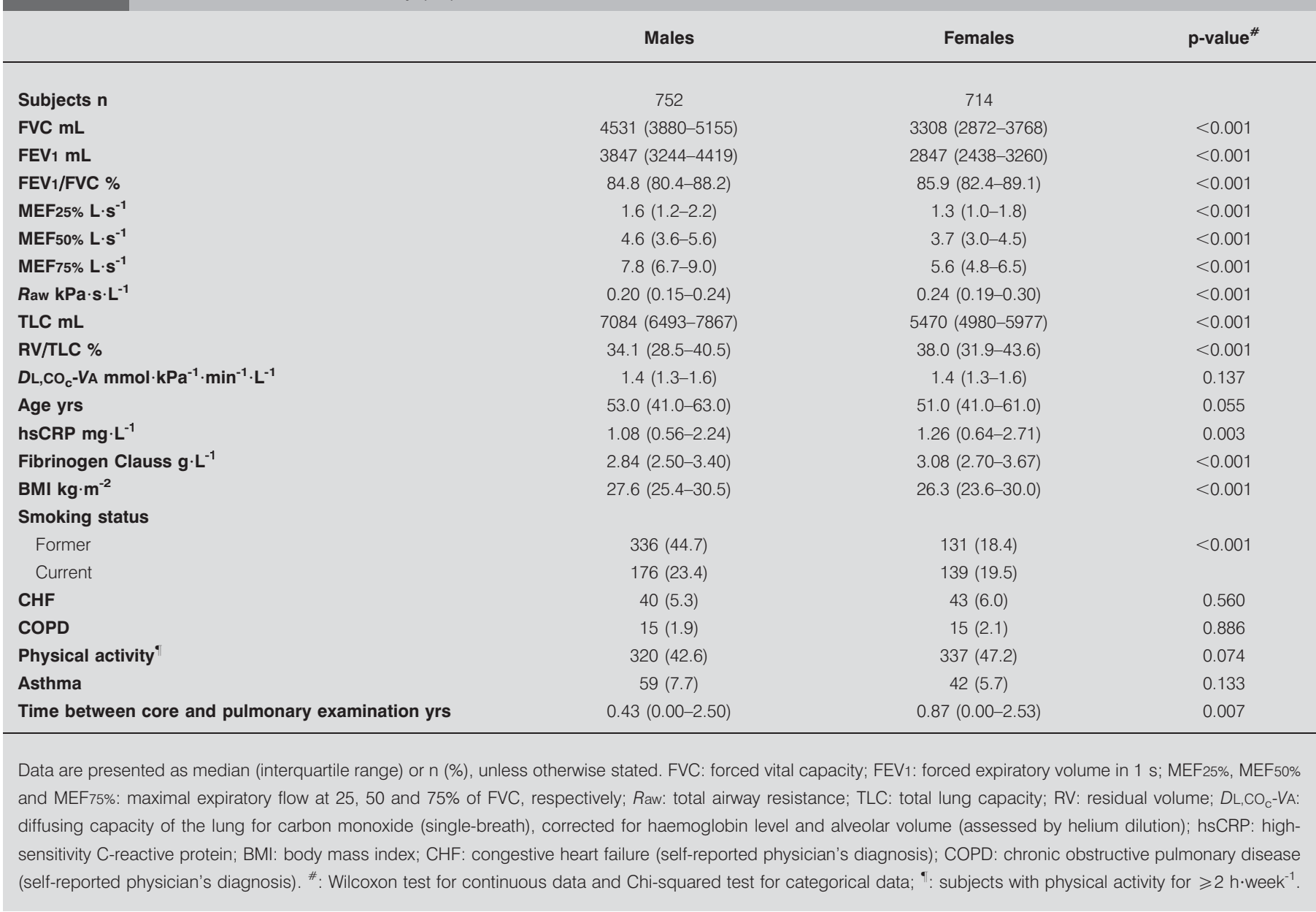


were performed to reduce potential bias due to missing values in complete case analyses [25]. Imputations were performed by the imputation by chained equations (ICE) procedure with 20 runs [26]. In this imputation, parameters of lung function were predicted by age, sex, BMI, smoking status, chronic heart failure and physical activity. Afterwards, regression analyses were repeated using the imputed data.

Tests were considered statistically significant with a two-sided p-value of $<0.05$. All statistical analyses were performed using Stata 11.0 (Stata Corporation, College Station, TX, USA).

\section{RESULTS}

Table 1 shows the characteristics of the study population stratified by sex. Compared with females, males had significantly higher values of FVC, FEV1, FRCpleth and TLC but lower values of FEV1/FVC, Raw and RV/TLC, while $D \mathrm{~L}, \mathrm{CO}_{\mathrm{c}}-V \mathrm{~A}$ was comparable between sexes. Moreover, males were more often smokers and had a higher BMI, but had lower hsCRP and fibrinogen levels than females. The median (interquartile range) hsCRP was $1.17(0.56-2.7) \mathrm{mg} \cdot \mathrm{L}^{-1}$ in non- and ex-smokers and $1.2(0.6-2.49) \mathrm{mg} \cdot \mathrm{L}^{-1}$ in smokers. Increasing BMI was associated with increasing hsCRP levels (BMI $<25 \mathrm{~kg} \cdot \mathrm{m}^{-2}: 0.77$ $(0.4-1.7) \mathrm{mg} \cdot \mathrm{L}^{-1}$; BMI $25-30 \mathrm{~kg} \cdot \mathrm{m}^{-2}: 1.15(0.63-2.18) \mathrm{mg} \cdot \mathrm{L}^{-1}$; $\left.\mathrm{BMI}>30 \mathrm{~kg} \cdot \mathrm{m}^{-2}: 2.32(1.14-4.87) \mathrm{mg} \cdot \mathrm{L}^{-1}\right)$.

Comparing SHIP-0 and SHIP-1, a significant intercorrelation of both inflammatory markers was verified (Spearman correlation coefficients: hsCRP 0.62 and fibrinogen 0.69; both $\mathrm{p}<0.001$ ).

To test for sex differences in the association between inflammatory markers and pulmonary variables, we added the interaction term between fibrinogen/hsCRP and sex to the fully adjusted models. These analyses revealed significant interaction terms for $\mathrm{DL}, \mathrm{CO}_{\mathrm{c}}-V \mathrm{~A}$ only. Consequently, regression analyses were performed in a sex-stratified manner for these variables, but not for the others.

Table 2 shows hsCRP and fibrinogen comparisons between subjects with normal and abnormal lung function (defined as central airflow limitation, lung volume restriction and DL,CO decrease). Subjects with central airflow limitation did not show different levels of hsCRP and fibrinogen, independent of the method of defining airflow limitation (FEV1/FVC and/or FEV1/VCslow ratios below the fifth percentile $(4.6 \%$ and $5.8 \%$ of the population, respectively)). The significant increase in hsCRP in subjects with $D \mathrm{~L}, \mathrm{CO}_{\mathrm{c}}-V \mathrm{~A}$ below the fifth percentile was completely attributable to male smokers; the increase in fibrinogen was found in smokers of both sexes. Applying the reference equations formulated by QUANJER et al. [23] for TLC confirmed the results, whereas using the reference equations for DL,CO derived by COTES et al. [24] did not show statistical significance.

Table 3 shows the results of the multivariable regression analyses. In the entire population, hsCRP was significantly associated with FVC, FEV1, MEF25\% and TLC, and fibrinogen was associated with FVC and FEV1. Furthermore, hsCRP and fibrinogen were associated with $\mathrm{DL}, \mathrm{CO}_{\mathrm{c}}-\mathrm{VA}$ in males; fibrinogen showed this association in females too (figs 1, 2 and 3). Data imputation confirmed associations of hsCRP with FEV1, TLC and $D L, \mathrm{CO}_{\mathrm{c}}-V \mathrm{~A}$ in males; for fibrinogen, association with $D \mathrm{~L}, \mathrm{CO}_{\mathrm{c}}-V \mathrm{~A}$ was confirmed in both sexes.

\begin{tabular}{|c|c|c|c|}
\hline \multirow[t]{2}{*}{ TABLE 2} & \multicolumn{3}{|c|}{$\begin{array}{l}\text { Comparison of subjects with and without } \\
\text { restrictive lung disease, airflow limitation and } \\
\text { diffusing abnormalities }\end{array}$} \\
\hline & $\geqslant 5$ th percentile ${ }^{\#}$ & $<5$ th percentile ${ }^{\#}$ & p-value \\
\hline \multicolumn{4}{|l|}{ hsCRP $\mathrm{mg} \cdot \mathrm{L}^{-1}$} \\
\hline FVC & $1.14(0.59-2.49)$ & $1.72(0.75-3.75)$ & $<0.001$ \\
\hline $\mathrm{FEV}_{1} / \mathrm{FVC}$ & $1.21(0.60-2.67)$ & $1.63(0.69-3.73)$ & 0.132 \\
\hline FEV $1 / N C$ slow & $1.23(0.61-2.70)$ & $1.02(0.53-2.62)$ & 0.446 \\
\hline TLC & $1.18(0.60-2.63)$ & $1.99(0.87-5.21)$ & $<0.001$ \\
\hline $\mathrm{TLC}^{+}$ & $1.21(0.60-2.66)$ & $1.70(0.83-4.07)$ & 0.028 \\
\hline$D \mathrm{~L}, \mathrm{CO}_{\mathrm{C}}-\mathrm{VA}_{\mathrm{A}}$ & $1.18(0.60-2.66)$ & $1.47(0.79-3.26)$ & 0.025 \\
\hline \multicolumn{4}{|c|}{ Fibrinogen $\mathbf{g} \cdot \mathrm{L}^{-1}$} \\
\hline FVC & $2.97(2.59-3.50)$ & $3.27(2.80-3.89)$ & $<0.001$ \\
\hline $\mathrm{FEV}_{1} / \mathrm{FVC}$ & $3.00(2.60-3.55)$ & $3.25(2.60-3.85)$ & 0.062 \\
\hline FEV 1 /NCslow & $3.00(2.60-3.60)$ & $2.81(2.50-3.50)$ & 0.192 \\
\hline TLC & $3.00(2.60-3.54)$ & $3.20(2.70-3.90)$ & 0.050 \\
\hline $\mathrm{TLC}^{+}$ & $3.00(2.60-3.58)$ & $3.11(2.57-3.80)$ & 0.527 \\
\hline$D \mathrm{~L}, \mathrm{CO}_{\mathrm{C}}-\mathrm{VA}_{\mathrm{A}}$ & $3.00(2.60-3.51)$ & $3.26(2.63-4.00)$ & 0.002 \\
\hline \multicolumn{4}{|c|}{ 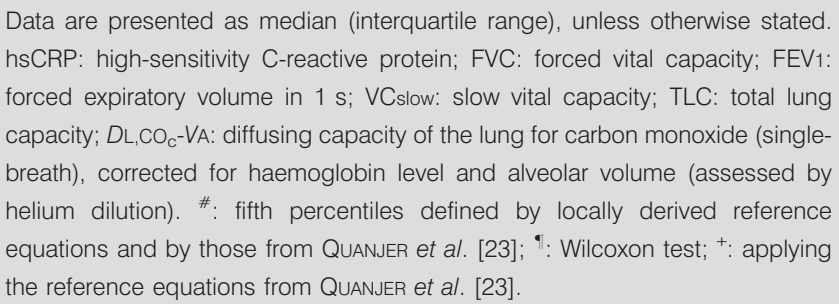 } \\
\hline
\end{tabular}

For sensitivity analysis, we excluded, in a stepwise manner, all participants with evidence of ischaemic heart disease (history of myocardial infarction, a pacemaker or electrocardiographic evidence of ischaemia), abnormal airflow limitation and restrictive lung disease. In this apparently healthy subgroup, no significant associations between lung volumes and inflammation were seen, independent of the applied reference equation for TLC. The stepwise exclusion revealed a major influence of TLC on the shown associations in the overall population. Substituting or adding height and/or weight and/or waist circumference did not alter the results substantially. In the subgroup of subjects with airflow limitation (FEV1/FVC and/or FEV1/VCslow ratios below the fifth percentile), hsCRP was significantly associated with $D \mathrm{~L}, \mathrm{CO}_{\mathrm{c}}-V \mathrm{~A}$ in males only $(\beta=0.4$, $95 \%$ CI $0.12-0.69 ; \mathrm{p}<0.01)$, but no other associations were verifiable.

\section{DISCUSSION}

In terms of the hypothesis framing this study, systemic inflammation quantified by serum hsCRP and fibrinogen levels is not associated with the presence of airflow limitation in central airways, as inferred from FEV1/FVC, FEV1/VCslow and Raw in a general population. In adults aged $25-85$ yrs, serum hsCRP is significantly associated with lung volumes as quantified by FEV1 and TLC. In smokers only, DL,CO is associated with hsCRP and fibrinogen. These associations were independent of cardiorespiratory fitness, lung diseases, cardiac diseases, obesity and smoking and (based on data imputations) are representative of the general population. 
TABLE 3 Associations between high-sensitivity C-reactive protein (hSCRP), fibrinogen and pulmonary variables

\begin{tabular}{|c|c|c|c|c|c|c|}
\hline & \multicolumn{2}{|c|}{ Whole population" } & \multicolumn{2}{|c|}{ Disease-free population } & \multicolumn{2}{|c|}{ Imputed data ${ }^{+}$} \\
\hline & Transformation $^{\S}$ & $\beta(95 \% \mathrm{Cl})$ & Transformation $^{\S}$ & $\beta(95 \% \mathrm{Cl})$ & Transformation $^{\S}$ & $\beta(95 \% \mathrm{Cl})$ \\
\hline \multirow[t]{2}{*}{ FVC mL } & $\log (\mathrm{hsCRP})$ & $-56.91(-91.74--22.08)^{\star \star \star}$ & hsCRP $^{1}$ & $-11.19(-30.96-8.57)$ & $\log (\mathrm{hsCRP})$ & $-42.98(-87.84-1.88)$ \\
\hline & Fibrinogen $^{1}$ & $-60.08(-109.03--11.13)^{*}$ & Fibrinogen $^{1}$ & $-36.14(-94.25-21.97)$ & Fibrinogen ${ }^{1}$ & $-43.74(-111.28-23.81)$ \\
\hline FEV $1 \mathrm{~mL}$ & $\log (\mathrm{hsCRP})$ & $-60.28(-90.92--29.64)^{\star \star \star}$ & $\mathrm{hsCRP}^{1}$ & $-9.98(-26.64-6.69)$ & $\log (\mathrm{hsCRP})$ & $-43.66(-82.98--4.34)^{*}$ \\
\hline FEV $1 /$ FVC $\%$ & Fibrinogen $^{1}$ & $-0.11(-0.60-0.38)$ & Fibrinogen $^{1}$ & $0.18(-0.32-0.69)$ & Fibrinogen ${ }^{1}$ & $-0.03(-0.52-0.47)$ \\
\hline \multirow[t]{2}{*}{ FEV $1 /$ VCslow } & hsCRP ${ }^{1}$ & $-0.00(-0.00-0.00)$ & hsCRP $^{1}$ & $-0.00(-0.00-0.00)$ & hsCRP $^{1}$ & $-0.00(-0.00-0.00)$ \\
\hline & Fibrinogen ${ }^{1}$ & $-0.00(-0.01-0.00)$ & Fibrinogen ${ }^{1}$ & $-0.00(-0.00-0.01)$ & Fibrinogen ${ }^{1}$ & $-0.00(-0.01-0.00)$ \\
\hline \multirow[t]{2}{*}{ MEF $25 \% L \cdot s^{-1}$} & $\mathrm{hsCRP}^{1}$ & $0.09(0.03-0.15)^{\star}$ & hsCRP-0.5 & $0.07(-0.00-0.14)$ & $\mathrm{hsCRP}^{1}$ & $0.05(-0.02-0.13)$ \\
\hline & Fibrinogen $^{1}$ & $-0.03(-0.07-0.02)$ & Fibrinogen $^{1}$ & $-0.00(-0.06-0.05)$ & Fibrinogen ${ }^{1}$ & $-0.01(-0.06-0.04)$ \\
\hline MEF $50 \% L \cdot s^{-1}$ & $\mathrm{hsCRP}^{1}$ & $-0.02(-0.05-0.01)$ & hsCRP $^{1}$ & $-0.00(-0.04-0.04)$ & hsCRP $^{1}$ & $0.00(-0.03-0.04)$ \\
\hline \multirow[t]{2}{*}{ FRCpleth $\mathrm{mL}$} & hsCRP ${ }^{1}$ & $-0.00(-0.01-0.01)$ & hsCRP ${ }^{1}$ & $-0.00(-0.03-0.02)$ & hsCRP $^{1}$ & $-0.00(-0.01-0.01)$ \\
\hline & Fibrinogen $^{1}$ & $-0.00(-0.06-0.06)$ & Fibrinogen ${ }^{1}$ & $-0.01(-0.08-0.07)$ & Fibrinogen $^{1}$ & $-0.01(-0.09-0.07)$ \\
\hline \multirow[t]{2}{*}{ Raw $\mathrm{kPa} \cdot \mathrm{s} \cdot \mathrm{L}^{-1}$} & $\mathrm{hsCRP}^{1}$ & $0.00(-0.00-0.00)$ & $\mathrm{hsCRP}^{1}$ & $-0.00(-0.00-0.00)$ & $\mathrm{hsCRP}^{1}$ & $0.00(-0.00-0.00)$ \\
\hline & Fibrinogen $^{1}$ & $0.00(-0.00-0.01)$ & Fibrinogen $^{1}$ & $-0.00(-0.01-0.01)$ & Fibrinogen ${ }^{1}$ & $0.00(-0.01-0.01)$ \\
\hline \multirow[t]{2}{*}{ TLC mL } & $\mathrm{hsCRP}^{3}$ & $-0.59(-0.95--0.24)^{*}$ & hsCRP $^{1}$ & $-24.85(-52.98-3.28)$ & hsCRP 3 & $-0.42(-0.80--0.05)^{*}$ \\
\hline & Fibrinogen $^{1}$ & $-52.10(-125.26-21.07)$ & Fibrinogen ${ }^{1}$ & $-40.78(-123.62-42.06)$ & Fibrinogen $^{1}$ & $-53.84(-141.19-33.52)$ \\
\hline \multirow[t]{2}{*}{$\mathrm{RV} / \mathrm{TLC} \%$} & hsCRP $^{1}$ & $-0.01(-0.20-0.18)$ & $\mathrm{hsCRP}^{1}$ & $-0.07(-0.28-0.13)$ & hsCRP $^{1}$ & $-0.15(-0.35-0.04)$ \\
\hline & Fibrinogen $^{1}$ & $0.52(-0.04-1.07)$ & Fibrinogen $^{1}$ & $0.22(-0.38-0.83)$ & Fibrinogen ${ }^{1}$ & $0.34(-0.27-0.95)$ \\
\hline \multirow{4}{*}{$\begin{array}{l}\text { DL, } \mathrm{CO}_{\mathrm{c}}-V_{\mathrm{A}} \text { males } \\
\mathrm{mmol} \cdot \mathrm{kPa}^{-1} \cdot \mathrm{min}^{-1} \cdot \mathrm{L}^{-1} \\
\mathrm{DL}, \mathrm{CO}_{\mathrm{c}}-V_{\mathrm{A}} \text { females } \\
\mathrm{mmol} \cdot \mathrm{kPa}^{-1} \cdot \mathrm{min}^{-1} \cdot \mathrm{L}^{-1}\end{array}$} & $\log ($ hsCRP) & $-0.06(-0.09--0.04)^{\star \star *}$ & $\mathrm{hsCRP}^{1}$ & $-0.01(-0.03-0.00)$ & $\log (\mathrm{hsCRP})$ & $-0.05(-0.07--0.02)^{\star \star \star}$ \\
\hline & Fibrinogen $^{1}$ & $-0.05(-0.09--0.02)^{*}$ & Fibrinogen $^{1}$ & $-0.03(-0.08-0.01)$ & Fibrinogen $^{1}$ & $-0.05(-0.09--0.02)^{\star}$ \\
\hline & hsCRP $^{1}$ & $-0.00(-0.01-0.01)$ & $\mathrm{hsCRP}^{1}$ & $-0.00(-0.02-0.01)$ & hsCRP $^{1}$ & $-0.01(-0.02-0.00)$ \\
\hline & Fibrinogen $^{1}$ & $-.04(-0.08--0.01)^{*}$ & Fibrinogen ${ }^{1}$ & $-0.02(-0.06-0.02)$ & Fibrinogen $^{1}$ & $-0.05(-0.09--0.01)^{\star}$ \\
\hline
\end{tabular}

Our study further strengthens previously described associations of inflammatory markers and lung volumes [1-4]. Available evidence clearly shows associations of spirometrically assessable and, thus, mobile lung volumes. Both FEV1 and FVC may be influenced by at least three mechanisms: airflow limitation, anatomical lung volume and respiratory muscle function [27]. Our study adds information from body plethysmographic and, thus, partly immobile and anatomically determined lung volumes. TLC, a reliable measure of lung size, is strongly associated with hsCRP. The strongest association of systemic inflammation with lung volumes and airflow restriction in the peripheral airways (FEV1, FVC, MEF25\%) is shown for hsCRP at levels $<3 \mathrm{mg} \cdot \mathrm{L}^{-1}$, while the FEV1/FVC ratio, a measure of more central airflow limitation, is unaffected. Effects on TLC were detected at higher hsCRP levels. In contrast, associations of fibrinogen with FVC and FEV1 showed a proportional decline over the full range of fibrinogen levels, even if these associations were not confirmed by imputed data and may not be representative of the overall population. The described differences between hsCRP and fibrinogen associations may potentially be explained by differences in course and level of inflammatory responses. In contrast to fibrinogen, hsCRP is generally accepted to more rapidly adjust to inflammatory stimuli. Furthermore, CRP in its high-sensitivity determination potentially describes inflammation in the very low-grade range. Scientific evidence suggests some impact of cardiorespiratory fitness and BMI on the degree of systemic inflammation $[9,28]$. However, even after adjusting for these potential influencing factors, associations between inflammatory markers and lung volumes remained. Thus, it is likely that systemic inflammation may influence lung volumes independently of these important confounders. Both lung volumes and hsCRP levels underlie genetic variants and pleiotropic effects and potential interactions may be possible [29, 30]. As a potential result, one may postulate that other factors, such as fetal and postnatal growth, may influence the potency of 

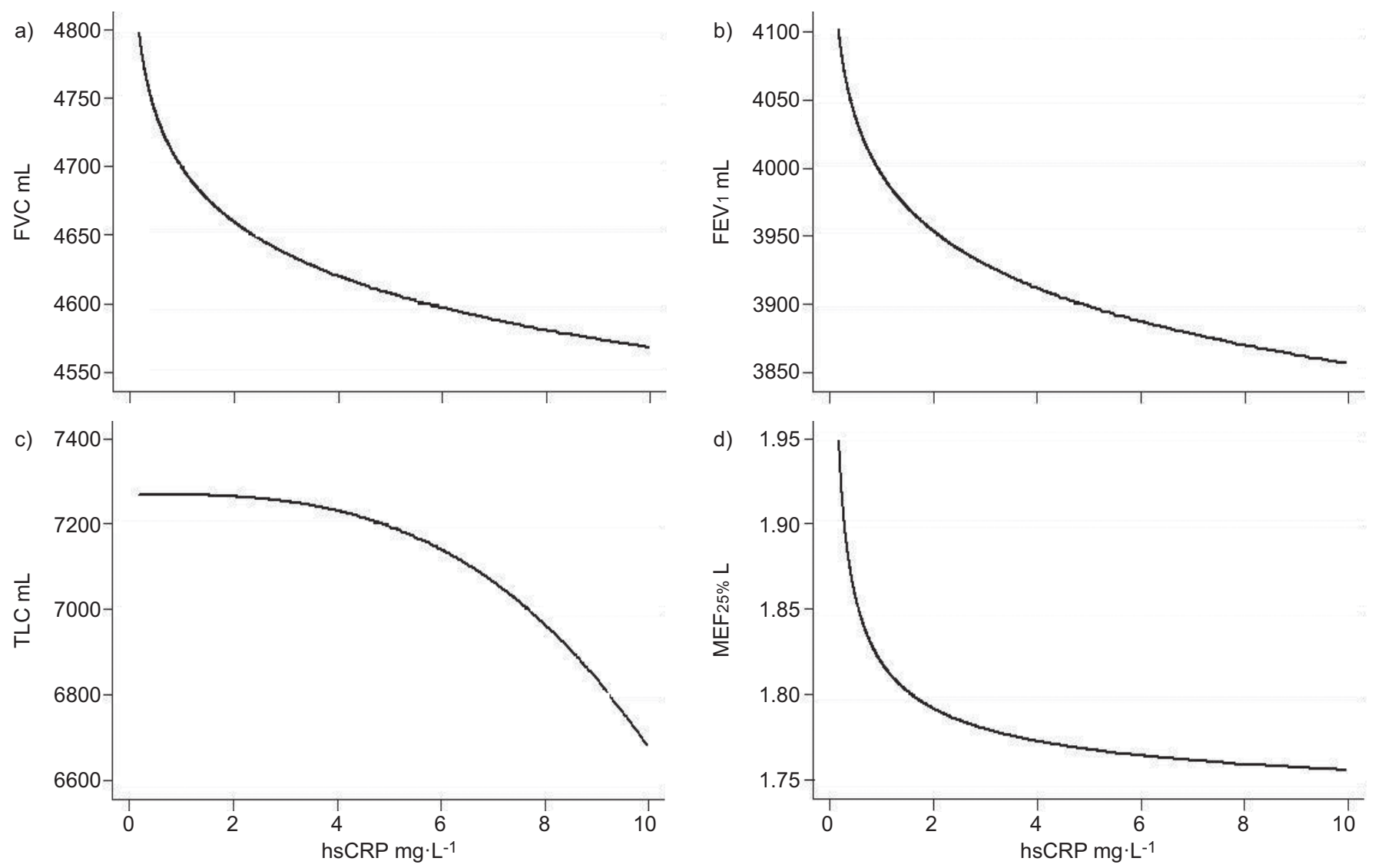

FIGURE 1. Significant associations between high-sensitivity C-reactive protein (hsCRP) and pulmonary variables in the overall population. a) Forced vital capacity (FVC), b) forced expiratory volume in $1 \mathrm{~s}$ (FEV1), c) total lung capacity (TLC) and d) maximal expiratory flow at 25\% of FVC (MEF25\%).

inflammatory responses, as well as affecting lung function development. This hypothesis is supported by the inverse association between birth weight and systemic inflammation [31], as well as by the association of low birth weight with

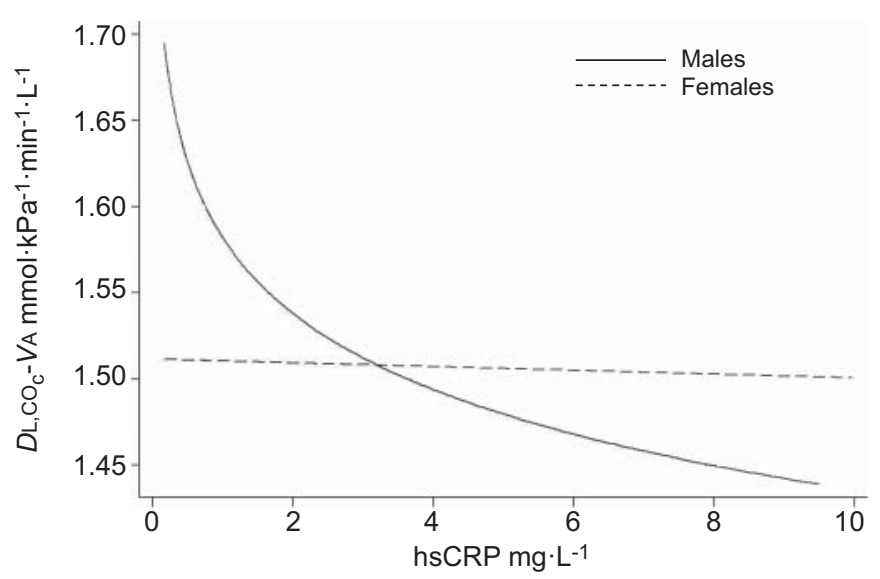

FIGURE 2. Significant associations between high-sensitivity C-reactive protein (hsCRP) and diffusing capacity of the lung for carbon monoxide (single-breath), corrected for haemoglobin level and alveolar volume (assessed by helium dilution) $\left(D L, \mathrm{CO}_{\mathrm{C}}-\mathrm{VA}\right)$, in the overall population. For this variable, hsCRP was significantly associated in males only. impaired lung volumes in adulthood [32]. However, this hypothesis has not yet been tested in a longitudinal observation.

Besides pulmonary anatomical structure and respiratory muscle strength, forced expiratory lung volumes are restricted by airflow limitation. Associations of inflammation with airflow limitation have been shown in selected populations suffering from COPD [8, 9]. Moreover, MELbYe et al. [33] reported significant associations between the degree of airflow limitation and blood CRP levels in a population sample of individuals aged $\geqslant 60$ yrs. With respect to airflow limitation in central airways, these data contrast with our results. Neither subjects with impaired more central airflow limitation defined by a FEV1/FVC or FEV1/VCslow ratio below the fifth percentile, nor healthy individuals, revealed any association between inflammation and airflow limitation (defined by FEV1/FVC or FEV1/ VCslow ratio and airway resistance). Correspondingly, pulmonary hyperinflation defined by the relation of RV to TLC was not associated with inflammatory markers. Interestingly, we found significant associations of MEF25\% and low-grade inflammation quantified by hsCRP. Even if this is small evidence, MEF25\% possibly reflects peripheral airway disease rather than central airflow limitation [34]. Thus, some influence of systemic inflammation on small airway function may be postulated, while central airways seem to be little affected. However, this association did not show robustness after data imputation and may not be representative for the overall SHIP population. 

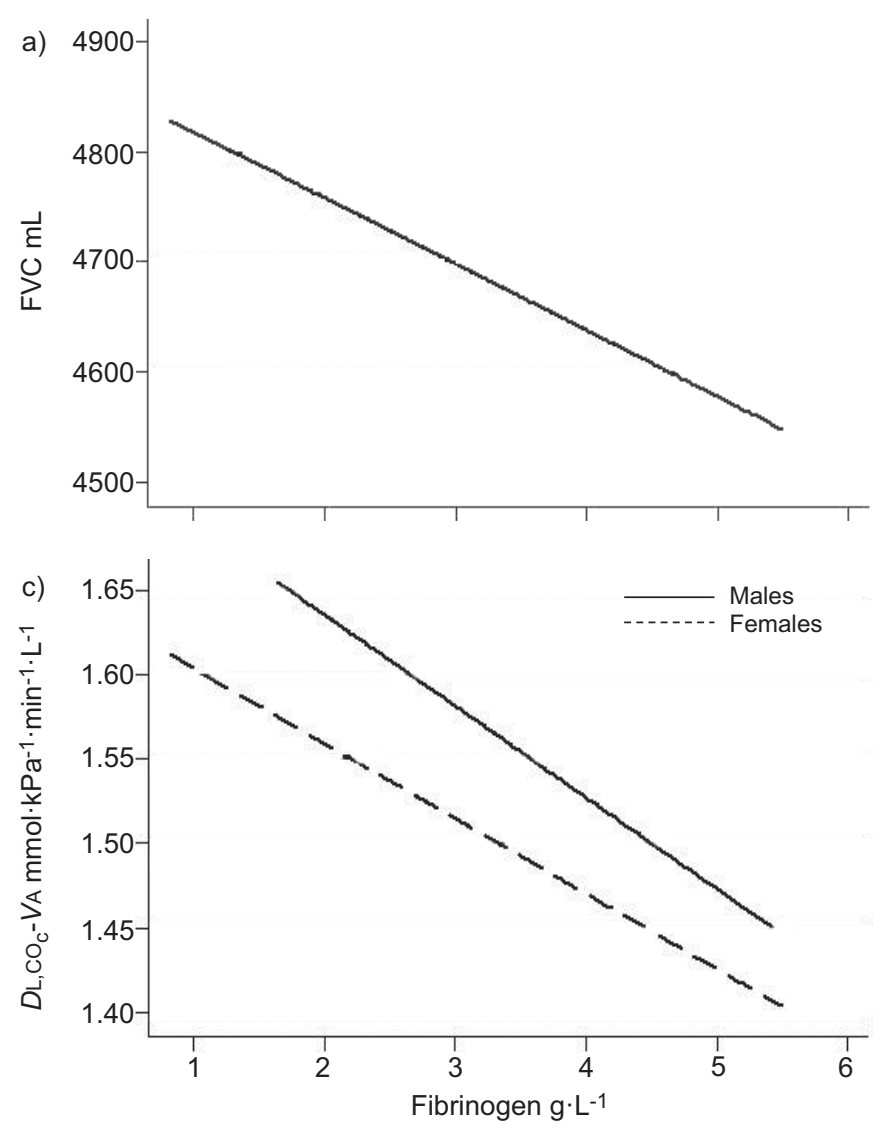

In comparison with previously published data, this study applies more accurate definitions of airflow limitation. As a potential measure of lung ageing, the FEV1/FVC ratio is substantially affected by age, resulting in an overestimation of airflow limitation in elderly individuals [35]. In addition, we applied the FEV1/VCslow ratio, as suggested by national authorities, resulting in a higher proportion of subjects with airflow limitation [36]. Thus, we assume that applying a more accurate definition of airflow limitation based on adjusted lower limits of normalcy and airway resistance is of substantial importance.

In smokers only, hsCRP and fibrinogen are significantly associated with DL,CO, suggesting a smoking-specific interaction. DL,CO is mainly affected by membrane and pulmonary vascular contributions [17]. In addition, ventilation-volume inhomogeneities [37] and ventilation-perfusion mismatching potentially affects $D L, C O$ [38]. The reasons behind the links between smoking, inflammation and impaired $D \mathrm{~L}, \mathrm{CO}$ may be given by the observation that, predominantly, perfusion heterogeneity and low-grade airflow obstruction are observed in early stages of COPD, suggesting that in smokers, the smallest airways, parenchyma and pulmonary vessels are affected initially [38]. Moreover, there is scientific evidence that smoking will induce endothelial dysfunction in pulmonary vessels, resulting in pulmonary vasoconstriction and decreased pulmonary blood volume [39]. To what extent one or more of these pathophysiological mechanisms may contribute to the described associations remains unclear. None of the associations focused on $D \mathrm{~L}, \mathrm{CO}$ could be confirmed by applying previously published

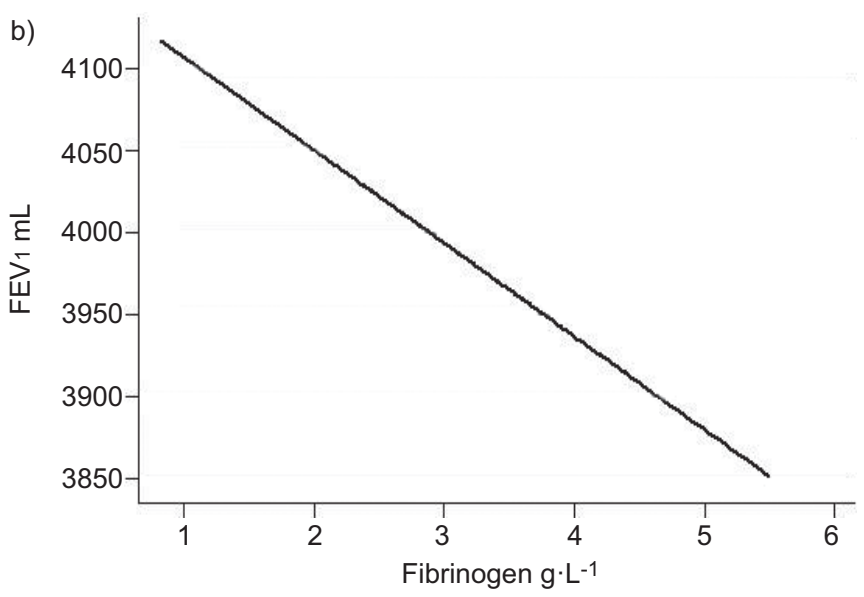

FIGURE 3. Significant associations between fibrinogen and pulmonary variables in the overall population. a) Forced vital capacity (FVC), b) forced expiratory volume in $1 \mathrm{~s}(\mathrm{FEV} 1)$ and $\mathrm{c}$ ) diffusing capacity of the lung for carbon monoxide (single-breath), corrected for haemoglobin level and alveolar volume (assessed by helium dilution) $\left(D \mathrm{~L}, \mathrm{CO}_{\mathrm{C}}-V_{\mathrm{A}}\right)$.

reference equations [24]. Even if this study was neither designed nor powered to clarify this hypothesis, this further underlines the proposed ERS recommendation of applying regionally derived reference equations [22].

The major strengths of this study are: the population-based approach representing a general adult population; the highly standardised fashion of obtaining lung function, including body plethysmography and DL,CO; and the adjustment for potential interfering factors. This study may be criticised for having a potential selection bias, but such a bias is almost inevitable in large-scale population-based studies. However, as the collected values were adjusted for numerous interfering factors, the differences between the general German population, the entire SHIP population and the lung function participants should be negligible. In addition, to minimise a potential recruitment bias, all associations have been re-assessed by imputed data. Based on data impution, associations of hsCRP and lung volumes (FEV1 and TLC) and DL,CO in males remained representative of the overall population; the same was the case for the fibrinogen- $D L, C O$ interaction. However, as usual in cross-sectional population-based investigations, all measures are based on an assessment at a single point in time. The basic principle of the study is not designed to discover pathophysiological mechanisms. For example, it remains impossible to prove to what extent lung function affects inflammation or vice versa. Furthermore, there are likely to be additional factors interfering with inflammation and lung function that were possibly not measured. However, according to previously published literature, known 
interfering factors for both lung function and inflammation have been included in the analyses [28].

\section{Conclusion}

In conclusion, a significant association of hsCRP as a surrogate of systemic inflammation and lung volumes in a general population has been identified, independently of obesity, age, sex, fitness or smoking. Central airflow limitation is not associated with systemic inflammation after adjustment for potential interfering factors, while peripheral lung structures may be involved. Further studies are needed to investigate the interaction of smoking, DL,CO and inflammation.

\section{SUPPORT STATEMENT}

The Study of Health in Pomerania (SHIP) is part of the Community Medicine Net of the University of Greifswald (Greifswald, Germany), which is funded by grants from the German Federal Ministry of Education and Research (BMBF) for SHIP (grants 01ZZ96030 and 01ZZ0701) and German Asthma and Chronic Obstructive Pulmonary Disease Network (COSYCONET; BMBF grant 01GI0883), the Ministry for Education, Research, and Cultural Affairs, and the Ministry for Social Affairs of the Federal State of Mecklenburg-West Pomerania.

\section{STATEMENT OF INTEREST}

None declared.

\section{REFERENCES}

1 Hancox RJ, Poulton R, Greene JM, et al. Systemic inflammation and lung function in young adults. Thorax 2007; 62: 1064-1068.

2 Rasmussen F, Mikkelsen D, Hancox RJ, et al. High-sensitive Creactive protein is associated with reduced lung function in young adults. Eur Respir J 2009; 33: 382-388.

3 Thyagarajan B, Jacobs DR, Apostol GG, et al. Plasma fibrinogen and lung function: the CARDIA Study. Int J Epidemiol 2006; 35: 1001-1008.

4 Yende S, Waterer GW, Tolley EA, et al. Inflammatory markers are associated with ventilatory limitation and muscle dysfunction in obstructive lung disease in well functioning elderly subjects. Thorax 2006; 61: 10-16.

5 Tice JA, Browner W, Tracy RP, et al. The relation of C-reactive protein levels to total and cardiovascular mortality in older US females. Am J Med 2003; 114: 199-205.

6 Beaty $\mathrm{TH}$, Cohen $\mathrm{BH}$, Newill CA, et al. Impaired pulmonary function as a risk factor for mortality. Am J Epidemiol 1982; 116: 102-113.

7 Lange P, Nyboe J, Appleyard M, et al. Spirometric findings and mortality in never-smokers. J Clin Epidemiol 1990; 43: 867-873.

8 Thorleifsson SJ, Margretardottir OB, Gudmundsson G, et al. Chronic airflow obstruction and markers of systemic inflammation: results from the BOLD study in Iceland. Respir Med 2009; 103: 1548-1553.

9 Watz H, Waschki B, Kirsten A, et al. The metabolic syndrome in patients with chronic bronchitis and COPD: frequency and associated consequences for systemic inflammation and physical inactivity. Chest 2009; 136: 1039-1046.

10 Volzke H, Alte D, Schmidt CO, et al. Cohort profile: the Study of Health in Pomerania. Int J Epidemiol 2011; 40: 294-307.

11 Spitzer C, Barnow S, Volzke H, et al. Association of posttraumatic stress disorder with low-grade elevation of C-reactive protein: evidence from the general population. J Psychiatr Res 2010; 44: 15-21.

12 World Health Organization. Guidelines for ATC Classification and DDD Assignment. Oslo, WHO Collaborating Centre for Drug Statistics Methodology, 2008.
13 Clauss A. Gerinnungsphysiologische Schnellmethode zur Bestimmung des Fibrinogens [Rapid physiological coagulation method in determination of fibrinogen]. Acta Haematol 1957; 17: 237-246.

14 Nelson SB, Gardner RM, Crapo RO, et al. Performance evaluation of contemporary spirometers. Chest 1990; 97: 288-297.

15 Wanger J, Clausen JL, Coates A, et al. Standardisation of the measurement of lung volumes. Eur Respir J 2005; 26: 511-522.

16 Miller MR, Hankinson J, Brusasco V, et al. Standardisation of spirometry. Eur Respir J 2005; 26: 319-338.

17 Macintyre N, Crapo RO, Viegi G, et al. Standardisation of the single-breath determination of carbon monoxide uptake in the lung. Eur Respir J 2005; 26: 720-735.

18 Royston P, Sauerbrei W. Multivariable Model-Building: A Pragmatic Approach to Regression Analysis based on Fractional Polynomials for Modelling Continuous Variables. Hoboken, John Wiley \& Sons, 2008.

19 Bleumink GS, Knetsch AM, Sturkenboom MC, et al. Quantifying the heart failure epidemic: prevalence, incidence rate, lifetime risk and prognosis of heart failure. The Rotterdam Study. Eur Heart J 2004; 25: 1614-1619.

20 Koch B, Schaper C, Ewert R, et al. Lung function reference values in different German populations. Respir Med 2010; 105: 352-362.

21 Koch B, Schaper C, Ittermann $T$, et al. Referenzwerte fur die Spirometrie bei Erwachsenen [Reference values for lung function testing in adults - results from the Study of Health in Pomerania (SHIP)]. Dtsch Med Wochenschr 2009; 134: 2327-2332.

22 Pellegrino R, Viegi G, Brusasco V, et al. Interpretative strategies for lung function tests. Eur Respir J 2005; 26: 948-968.

23 Quanjer PH, Tammeling GJ, Cotes JE, et al. Lung volumes and forced ventilatory flows. Report Working Party Standardization of Lung Function Tests, European Community for Steel and Coal. Official Statement of the European Respiratory Society. Eur Respir J 1993; 6: Suppl. 16, 5-40.

24 Cotes JE, Chinn DJ, Quanjer PH, et al. Standardization of the measurement of transfer factor (diffusing capacity). Report Working Party Standardization of Lung Function Tests, European Community for Steel and Coal. Official Statement of the European Respiratory Society. Eur Respir J 1993; 6: Suppl. 16, 41-52.

25 van Buuren S, Boshuizen HC, Knook DL. Multiple imputation of missing blood pressure covariates in survival analysis. Stat Med 1999; 18: 681-694.

26 Sterne JA, White IR, Carlin JB, et al. Multiple imputation for missing data in epidemiological and clinical research: potential and pitfalls. BMJ 2009; 338: b2393.

27 Standardization of spirometry - 1987 update. Official statement of American Thoracic Society. Respir Care 1987; 32: 1039-1060.

28 Aronson D, Avizohar O, Levy Y, et al. Factor analysis of risk variables associated with low-grade inflammation. Atherosclerosis 2008; 200: 206-212.

29 Dehghan A, Dupuis J, Barbalic M, et al. Meta-analysis of genomewide association studies in $>80,000$ subjects identifies multiple loci for C-reactive protein levels. Circulation 2011; 123: 731-738.

30 Repapi E, Sayers I, Wain LV, et al. Genome-wide association study identifies five loci associated with lung function. Nat Genet 2010; 42: 36-44.

31 Sattar N, McConnachie A, O'Reilly D, et al. Inverse association between birth weight and C-reactive protein concentrations in the MIDSPAN Family Study. Arterioscler Thromb Vasc Biol 2004; 24: 583-587.

32 Barker DJ, Godfrey KM, Fall C, et al. Relation of birth weight and childhood respiratory infection to adult lung function and death from chronic obstructive airways disease. BMJ 1991; 303: 671-675.

33 Melbye H, Halvorsen DS, Hartz I, et al. Bronchial airflow limitation, smoking, body mass index, and statin use are strongly associated with the C-reactive protein level in the elderly. The Tromso Study 2001. Respir Med 2007; 101: 2541-2549. 
34 Koulouris NG, Dimopoulou I, Valta P, et al. Detection of expiratory flow limitation during exercise in COPD patients. J Appl Physiol 1997; 82: 723-731.

35 Hansen JE, Sun XG, Wasserman K. Spirometric criteria for airway obstruction: use percentage of FEV1/FVC ratio below the fifth percentile, not $<70 \%$. Chest 2007; 131: 349-355.

36 Vogelmeier C, Buhl R, Criee CP, et al. Leitlinie der Deutschen Atemwegsliga und der Deutschen Gesellschaft für Pneumologie und Beatmungsmedizin zur Diagnostik und Therapie von Patienten mit chronisch obstruktiver Bronchitis und Lungenemphysem (COPD) [Guidelines for the diagnosis and therapy of COPD issued by Deutsche Atemwegsliga and Deutsche Gesellschaft fur Pneumologie und Beatmungsmedizin]. Pneumologie 2007; 61: e1-e40.

37 Verbanck S, Schuermans D, Van Muylem A, et al. Conductive and acinar lung-zone contributions to ventilation inhomogeneity in COPD. Am J Respir Crit Care Med 1998; 157: 1573-1577.

38 Rodriguez-Roisin R, Drakulovic M, Rodriguez DA, et al. Ventilationperfusion imbalance and chronic obstructive pulmonary disease staging severity. I Appl Physiol 2009; 106: 1902-1908.

39 Wang T, Han SX, Zhang SF, et al. Role of chymase in cigarette smoke-induced pulmonary artery remodeling and pulmonary hypertension in hamsters. Respir Res 2010; 11: 36. 